\title{
Enabling Atrial Fibrillation Detection using a Weight Scale
}

\author{
Brian Ayers ${ }^{1}$, Connor Beshaw ${ }^{1}$, Ernesto Serrano-Finetti ${ }^{2}$, Oscar Casas ${ }^{2}$, Ramon Pallas-Areny², \\ Jean-Philippe Couderc ${ }^{1}$
${ }^{1}$ Heart Research Follow-Up Program, Cardiology Department, University of Rochester Medical Center, Rochester, NY, USA
${ }^{2}$ Universitat Politècnica de Catalunya, BarcelonaTech, Barcelona, Spain

\begin{abstract}
Atrial fibrillation (AF) is a cardiac arrhythmia characterized by a highly irregular heart rate. It is the most prevalent arrhythmia in the general population in the United States and most developed countries, and is strongly associated with increased morbidity and mortality from adverse cardiovascular and cerebrovascular events. Moreover, patients with AF are correlated with increased healthcare expenditures, making the burden of AF on society extremely large.

In this proof-of-concept study, we present an innovative device to be used as a screening tool for AF. The device consists of a modified electronic scale that is able to obtain an ECG and analyse it for the presence of $A F$. The classification algorithm is based on the RdR map method that plots $R R$ intervals versus change in $R R$ intervals.

After optimizing the algorithm on a learning set of 77 ECGs from 45 patients, the performance of the device during a blind validation of 76 ECGs from 44 patients was: accuracy $=83 \%$, sensitivity $=83 \%$, specificity $=$ 83\% ( $N=76$ ECGs). Applying a constraint that each ECG recording contains a minimum of 7 beats in order to be eligible for classification, accuracy improved to $89 \%$ (sensitivity $=83 \%$, specificity $=90 \%, N=70$ ).

In conclusion, we present an innovative device to detect $A F$ in a manner that can be implemented into current physician workflow without increasing the time or cost of each clinical encounter.
\end{abstract}

\section{Introduction}

Atrial fibrillation (AF) is the most common persistent cardiac arrhythmia in the general population and is associated with increased morbidity, mortality and healthcare expenditures [1,2]. Currently an estimated 3 million people in the United States have AF, and this number is expected to more than double in the next 25 years with 1 in 4 adults 40 years and older developing AF during their lifetime [3]. AF is a potent risk factor for cardiovascular and cerebrovascular events, resulting in an elevated mortality risk after adjusting for pre-existing cardiovascular conditions [1].

Alarmingly, over $30 \%$ of individuals with AF are unaware of having the condition, putting them at significant risk for adverse outcomes [4]. In addition to health risks, AF is associated with higher healthcare costs as Medicare spending is 10 -fold greater in the first year for men and women with AF [2]. Given this substantial and increasing burden of $\mathrm{AF}$, an accurate and costeffective screening tool is needed.

In this study, we evaluate the ability of a modified weight scale to detect the presence of AF without the use of a standard ECG recording machine. Importantly, this novel device is designed to be seamlessly incorporated into physicians' current workflow, allowing for AF screening without any significant changes to current medical routines. In this proof-of-concept study we provide an assessment of the device's accuracy for detecting AF in subjects in a clinical setting.

\section{Methods}

\subsection{The ECG weight scale}

The "ECG Weight Scale" (EWS) is a prototype consisting of a simple commercially available electronic weight scale integrated with four sensors to measure the body surface ECG from the subject's limbs. Two of the sensors are attached to the top of the scale to allow for contact with the subject's feet and the other two sensors are designed to be held in the subject's hands while standing on the scale during weight measurement (Figure 1 , device in use in panel A) [5].

The four electrodes are connected to electronic circuits with a system bandwidth of $0.05 \mathrm{~Hz}$ to $100 \mathrm{~Hz}, 16$-bit resolution, and a $1 \mathrm{kHz}$ sampling rate for the recording and processing of the acquired signals $(333 \mathrm{~Hz}$ per lead signal). A laptop is connected via USB 3.0 port to deliver a $5.00 \mathrm{~V}$ power source and for visualization and storage 
of the data. The EWS was initially tested as a new device to measure the heart rate and QT/QTc intervals [5]. In this new endeavour, we planned to evaluate the ability of the EWS to detect the presence of AF.

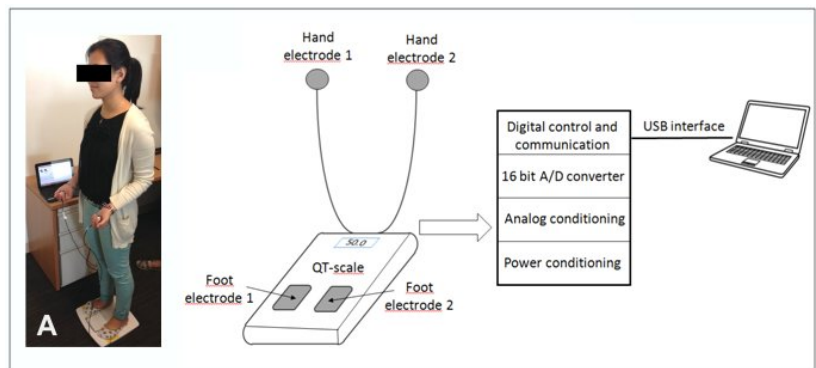

Figure 1. Schematic depiction of the components of the EWS. Panel A demonstrates the EWS in use while measuring a subject's weight and ECG simultaneously.

\subsection{Study population}

We present a preliminary evaluation of the ability of the EWS to detect the presence of $\mathrm{AF}$ in a cohort of cardiac patients going through an inpatient clinic (Clinical Cardiology Division at the University of Rochester Medical Center, Rochester, NY). This cohort includes patients with very diverse types of cardiac disease, including AF patients going through dofetilide protocol for drug-induced cardioversion of AF.

The patients going through the dofetilide protocol were in AF at the time of their first ECG recording. In these dofetilide subjects, three recording sessions were implemented per subject; Session 1: baseline recording prior to first dose of dofetilide; Session 2: recording prior to second dose of dofetilide; and Session 3: recording prior to hospital release. During each session, we acquired two ECGs in the standing position on the EWS. A total of 6 ECG recordings were acquired for each patient undergoing dofetilide protocol, while all other cardiac patients were asked to stand on the EWS twice to acquire 2 ECGs 1 minute apart during their visit. All subject ECGs were recorded for $30 \mathrm{~s}$ on the EWS.

The enrollment criteria specified that subjects are adult cardiac patients (age $\geq 18$ years), excluding patients that could not comply with the use of the weight scale including patients with muscular tremors and/or Parkinson disease. The protocol was approved by the University of Rochester (Rochester, NY) Research Review Board.

All ECG recordings were interpreted by two trained ECG experts who classified the recordings as representing either $\mathrm{AF}$ or normal sinus rhythm. One recording was excluded from the study because it was non-interpretable due to poor quality of the signal. The cohort data was randomly separated into two groups to generate a learning dataset and an independent validation dataset.

\subsection{AF detection}

Processing procedures for $\mathrm{R}$ peak detection were designed in a prior study involving healthy subjects only [unpublished data]. Based on the learning set of data, the resulting RR values were filtered to exclude extreme outliers that reflect inaccurate $\mathrm{R}$ peak detection. $\mathrm{RR}$ values were retained using the following criteria: $-400<$ $\mathrm{dRR}<800 \mathrm{~ms}$, and $200<\mathrm{RR}<1600 \mathrm{~ms}$ (38 to 300 bpm). This range was selected by visual inspection of all the data points in the learning set and their corresponding ECG tracings to determine extreme outliers that represent incorrect values (3\% of collected data points were excluded). The dRR constraints were set to eliminate these outliers, which predominately represent missed QRS complexes during $\mathrm{R}$ peak detection. The RR intervals were then analysed for AF according to a modified implementation of the previously described RdR map method [6].

The RdR map is constructed by plotting the $\mathrm{RR}$ interval series against the $d R R$ series $\left(d R R_{i}=R R_{i}-R R_{i}\right.$ 1). This allows for the separation of irregularly spaced heartbeats characteristic of AF from regularly paced beats associated with normal sinus rhythm (Figure 2).

To quantify variability in heart rate, the RdR map is divided into a two-dimensional grid. The number of data points within each cell of the grid is then counted. The total number of nonempty cells (NECs; i.e., cell contains $>=1$ data point) is then summed as a measure of the irregularity of the RR intervals. AF is expected to display a greater number of NEC than normal sinus pacing.

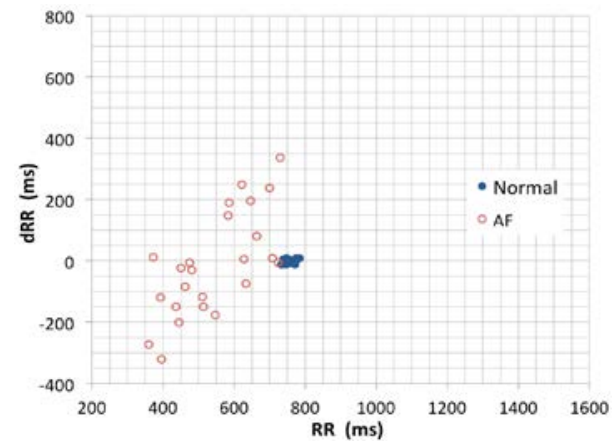

Figure 2. Representative examples of EWS device recordings for a subject in sinus rhythm (filled circles) and a subject in AF (open circles) using the RdR grid. The AF example demonstrates much more heartbeat variability $(\mathrm{NEC}=21)$ than the normal sinus rhythm $(\mathrm{NEC}=4)$.

We obtained ECG recordings with a variable number of cardiac beats depending on the subject's heart rate. To account for this inter-subject variability of recorded beats, we used the ratio of NEC to total number of beats per recording as our classification criteria ("NEC ratio"). Recordings were classified using a threshold for the NEC ratio. 
Based on the learning set of recordings, the resolution of the two-dimensional grid (cell size) and the NEC ratio threshold were optimized. This was achieved by systematically varying the cell size (10 - $100 \mathrm{~ms})$ and threshold (0.05 - 0.90) for maximizing classification accuracy of the learning set.

\subsection{Impact of EWS recording duration}

We investigated the role of ECG duration (number of beats) on the accuracy of the proposed algorithm. We used all ECG recordings from the learning set to analyze specific durations ranging from 2 to 24 beats. Longer recordings were truncated so that all examples contained the same or fewer numbers of beats than the proposed value. The cell size and NEC ratio threshold were held constant at the previously optimized values. Overall accuracy of the algorithm for each length was used to assess the relationship between the number of beats in a recording and the performance of the algorithm in classifying AF.

\section{Results}

\subsection{Study population:}

There were a total of 153 ECG recordings analyzed during this study. Of these, 25 were recorded during AF and 128 were in sinus rhythm. The recordings were randomly divided into learning and validation sets. Table 1 shows the patient characteristics of each dataset.

Table 1. Patient characteristics of the datasets.

\begin{tabular}{lcc}
\hline & Learning & Validation \\
\hline$N$ (ECGs) & 77 & 76 \\
$M$ (Patients) & 45 & 44 \\
Age (mean \pm std) & $62 \pm 12$ & $62 \pm 11$ \\
Gender (\% male) & $66 \%$ & $70 \%$ \\
Cohort (\% from cohort 1$)^{1}$ & $31 \%$ & $39 \%$ \\
\hline
\end{tabular}

${ }^{1}$ Cohort 1 consists of patients undergoing cardioversion

\subsection{Defining the AF detection procedure}

The learning set was used to investigate the optimal cell size and NEC ratio threshold for our modified RdR map method. Figure 3 describes the results of the investigation using a heat map in which lighter gray corresponds to low accuracies and darker gray to high levels of classification accuracies. The grid cells associated with $90 \%$ or higher accuracy are labeled.

We determined the optimal cell size to be $50 \mathrm{~ms}$ and NEC ratio threshold to be 0.75 (Figure 3). Using these parameters, the results obtained on the learning set data were an overall accuracy of $92 \%$ with sensitivity and specificity of $85 \%$ and $94 \%$, respectively ( $N=77$ ECGs).

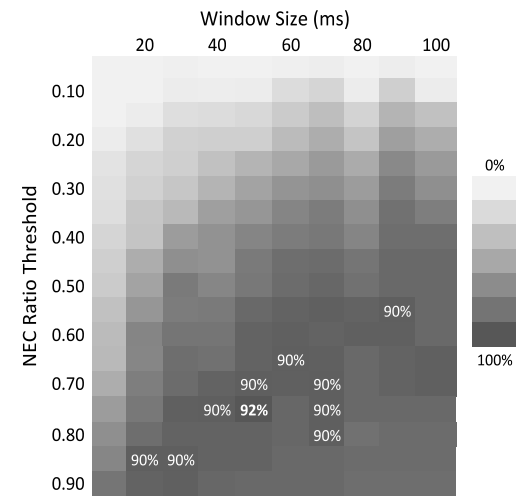

Figure 3. Cell size and NEC ratio values were selected on the learning set to maximize the accuracy in detecting AF. A cell size of $50 \mathrm{~ms}$ and NEC ratio threshold of 0.75 yielded the highest overall accuracy and were used for the validation set.

Holding the cell size and NEC ratio threshold constant at the previously optimized values, we found that classification accuracy is greatly diminished when considering recordings with less than 7 beats (Figure 4).

If we use this 7 beat threshold as a minimum number of beats required in an ECG recording to consider the recording for AF interpretation, then we get a $95 \%$ overall accuracy with sensitivity and specificity of $85 \%$ and $97 \%$, respectively ( $N=73$ ECGs). However, in applying this minimum number of beats constraint we remove $5 \%$ of the learning set cohort from consideration.

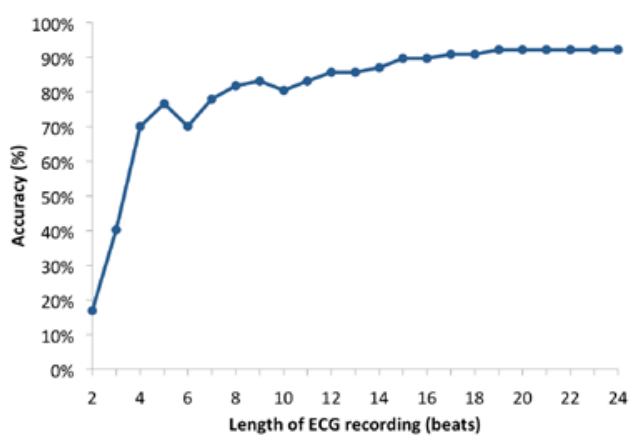

Figure 4. Describing the impact of ECG recording length on AF classification accuracy. Longer recordings were truncated so that the length of all examples considered was $<=$ given number of beats ( $X$ axis). Cell size and NEC ratio thresholds were held constant at $50 \mathrm{~ms}$ and 0.75 , respectively.

\subsection{Validation of the AF detection method}

The AF classification algorithm optimized on the learning set was applied to the validation set for a blinded validation. If no minimum number of beats were required for a recording to be considered, the method had an overall accuracy of $83 \%$ with similar sensitivity and specificity values of $83 \%(N=76$ ECGs $)$. Figure 5 displays the values of the NEC ratio for the recordings of the validation set. 
If we consider the requirement that an ECG recording must contain at least 7 beats in order to be considered, the overall accuracy improves to $89 \%$ with sensitivity and specificity of $83 \%$ and $90 \%$, respectively ( $N=70$ ECGs).

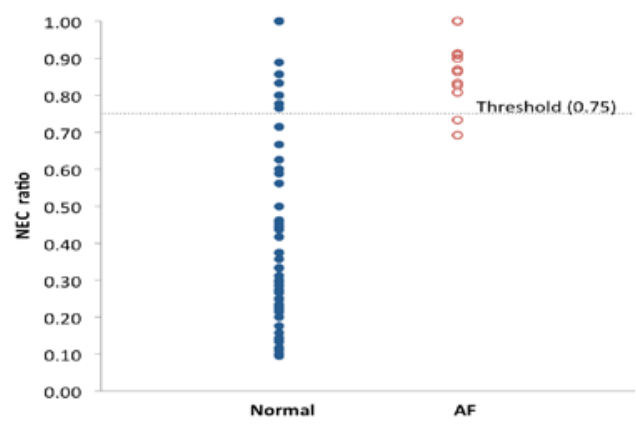

Figure 5. NEC ratio for 76 ECG recordings from the validation set. The NEC ratio threshold that was optimized on the learning set is depicted. Examples with NEC ratio $>0.75$ were classified as AF (11 false positives and 2 false negatives).

\section{Discussion}

This proof-of-concept study presents evidence of the capability of the EWS to be used to detect the presence of AF in a clinical setting. The EWS device is a standard weight scale with four embedded sensors that are able to obtain an ECG signal. Two of the sensors are attached to the top of the scale to allow for contact with the subject's feet and the other two sensors are designed to be held in the subject's hands during weight measurement. The device has previously been shown to be able to detect prolonged QT intervals [5].

After optimizing the parameters of the AF detection algorithm using a learning set, an overall accuracy of $83 \%$ was achieved on a blinded validation set (sensitivity = $83 \%$, specificity $=83 \%, N=76$ ECGs). While this study displays proof of concept of the device's potential, the performance of the technology has been limited by the length and quality of the ECG recordings.

First, the ECG recordings that were collected in this study are extremely short in length ( $30 \mathrm{~s}$ ) and therefore pose a challenge in quantifying the heart rate variability characteristic of AF rhythm. We purposefully limited the duration of the ECG recordings to minimize the impact the device would have on current physician workflow. While this approach shows promise, some recordings were unable to record a sufficient number of beats in order to enable AF detection. To address this, we plan to develop an EWS device that could deliver a functional assessment of the quality of the recorded signal in real time in order to determine the number of heartbeats captured. This real-time assessment could determine the utility of the recorded signal and indicate to the patient to remain on the weight scale until the ECG recording is of sufficient quality to ensure reliable detection of AF.
Further work will also be made to increase the continuity of contact between the sensors and the limbs of the patients, specifically the feet. The next version of the prototype will need to integrate larger sensors for the feet to ensure better continuous contact between the sensor and subject. This should reduce the variability in RR detection recorded in normal sinus rhythm patients, thereby decreasing the number of false positives classified as AF.

Increasing the specificity of AF classification will be instrumental in maximizing the device's utility in a clinical setting. In order to be cost effective, the device must minimize the number of costly false positives that would require further testing. Improving sensor contact with the subject and implementing real time assessment of the quality of the signal should increase specificity and make the EWS a clinically useful screening tool to combat the burden of AF on society.

\section{Conclusion}

We present an innovative device that enables the detection of AF in a clinical setting in a manner that fits seamlessly into current physician workflow without greatly increasing the time or cost of each clinical encounter.

\section{References}

[1] Benjamin EJ, Wolf PA, D'Agostino RB, Silbershatz H, Kannel WB, Levy D. Impact of atrial fibrillation on the risk of death: the Framingham Heart Study. Circulation 1998;98:946-52.

[2] Wolf PA, Mitchell JB, Baker CS, Kannel WB, D'Agostino RB. Impact of atrial fibrillation on mortality, stroke, and medical costs. Arch Intern Med 1998;158:229-34.

[3] Lloyd-Jones DM, Wang TJ, Leip EP, Larson MG, Levy D, Vasan RS, D'Agostino RB, Massaro JM, Beiser A, Wolf PA, Benjamin EJ. Lifetime risk for development of atrial fibrillation: the Framingham Heart Study. Circulation 2004;110:1042-6.

[4] Patten M, Maas R, Karim A, Müller HW, Simonovsky R, Meinertz T. Event-recorder monitoring in the diagnosis of atrial fibrillation in symptomatic patients: subanalysis of the SOPAT trial. J Cardiovasc Electrophysiol 2006;17:1216-20.

[5] Couderc JP, Beshaw C, Niu X, Serrano-Finetti E, Casas O, Pallas-Areny R, Rosero S, Zareba W. The QT Scale: A Weight Scale Measuring the QTc Interval, Annals of NonInvasive Electrocardiol 2016, submitted.

[6] Lian J, Wang L, Muessig D. A simple method to detect atrial fibrillation using RR intervals. Am J Cardiol 2011;107:1494-7.

Address for correspondence.

Brian Ayers

8 Eastbrook Farm Lane, Southborough, MA 01772

Brian_Ayers@URMC.Rochester.edu 